\title{
Inversão Conjunta de dados MMT 2D (modos TM e TE) de Ambientes Altamente Resistivos em Águas Profundas
}

Frayzer Lima de Almeida, UFPA, Brasil.

frayzerlima@hotmail.com

Copyright 2019, SBGf - Sociedade Brasileira de Geofísica

This paper was prepared for presentation during the $16^{\text {th }}$ International Congress of the Brazilian Geophysical Society held in Rio de Janeiro, Brazil, 19-22 August 2019.

Contents of this paper were reviewed by the Technical Committee of the $16^{\text {th }}$ International Congress of the Brazilian Geophysical Society and do not necessarily represent any position of the SBGf, its officers or members. Electronic reproduction or storage of any part of this paper for commercial purposes without the written consent of the Brazilian Geophysical Society is prohibited.

\section{Abstract}

We applied the geophysical inversion on synthetic data of the Marine Magnetotelluric 2D - MMT 2D method (TM and TE modes) derived from a highly resistive geoelectric model characteristic of the exploration of hydrocarbons in deep waters similar to the Brazilian coastal basins. The inversion method used (Marquardt method) uses the a priori introduction of absolute bonds and the inclusion, also a priori, of a given real range of absolute electrical resistivity. The inversion was applied individually on the synthetic data for both the TM mode and the TE mode, as well as on the TM and TE mode data of the MMT 2D. The synthetic data are obtained through the direct modeling of 2D MMT (TM and TE modes) according to the twodimensional finite element method and added with $7 \%$ of noise over apparent resistivities and $3 \%$ over impedance phases. The synthetic data are obtained through the direct modeling of 2D MMT (TM and TE modes) according to the two-dimensional finite element method and added with $7 \%$ of noise in the apparent resistivity data and $3 \%$ in the impedance phase data. The inversion results are presented and evaluated in terms of the absolute electrical resistivity.

\section{Introdução}

Os métodos marinho de fonte controlada - mCSEM e o magnetotelúrico marinho - MMT são métodos geofísicos eletromagnéticos que se destacam na prospecção de hidrocarbonetos em águas profundas e ultra-profundas, pois usados em conjunto com a sísmica podem confirmar a existência de hidrocarbonetos, mapear o contorno de um reservatório e contribuir para a diminuição da ambiguidade na interpretação geofísica.

O mCSEM não é capaz de mapear a base da estrutura salina (corpo altamente resistivo) localizado em águas profundas, devido suas medidas, de baixa frequência, serem afetadas pela variação de resistividade interna desta estrutura, juntamente com a sua profundidade. Por outro lado o imagiamento sísmico em profundidade apresenta baixa resolução na região abaixo da estrutura salina, um vez que, estas estruturas apresentam elevada velocidade de propagação sísmica em relação às rochas sedimentares circundantes, proporcionando a difração dos caminhos dos raios sísmicos e a diminuição da energia de propagação transferida para os sedimentos abaixo da estrutura salina (Hoversten, 2000). Porém, ambientes geológicos compostos por camada salina contendo em sua base uma camada reservatório com hidrocarbonetos, localizadas em águas profundas, e circundadas por sedimentos menos resistivos são melhores investigados através do método MMT, uma vez que este método eletromagnético é indicado para fornecer informações da distribuição de resistividade elétrica das rochas de subsuperfície, desde que exista significativa razão e/ou contraste de resistividade entre estas rochas.

Apresentamos o problema direto do método MMT 2D (modos TM e TE). Em seguida propomos a metodologia de inversão a ser utilizada sobre dados do MMT 2D para os modos TM e TE (Almeida, 2017). Em seguida, através de um modelo geoelétrico bidimensional de ambiente altamente resistivo localizado em águas profundas, mostramos os resultados de inversão bidimensional, segundo dados sintéticos do modo TM, do modo TE e dados sintéticos conjuntos dos modos TM e TE do MMT 2D.

\section{O Problema direto}

Os campos magnético e elétrico, secundários, podem ser expressos a partir das equações de Maxwell (no sistema SI) no domínio da frequência e em um meio não magnético (Rijo, 2002). Assim, sobre estas equações admite-se que suas resistividades variam apenas nas direções $z$ e $x$, originando dois sistemas de equações diferenciais parciais, os quais caracterizam modos distintos de propagação eletromagnética, isto é, o modo transversal magnético (modo TM) e modo transversal elétrico (modo TE). Tais sistemas de equações são solucionados, individualmente, através do método dos elementos finitos bidimensionais (Rijo, 2002) aplicando o critério de Galerkin (Becker; Carey; Oden, 1981). Quando se aplica o método dos elementos finitos bidimensionais sobre cada sistema acima mencionado, surge (para cada modo de propagação) um sistema linear associado a uma matriz complexa, simétrica e esparsa, em que cada sistema é solucionado numericamente pelo método do gradiente biconjugado (Souza et al., 2005). Sugerimos os trabalhos de Saito (2009) e Almeida (2015), para um entendimento detalhado da modelagem direta do MMT 2D.

\section{O problema inverso}

A função objetivo minimizada no presente trabalho é a proposta por Almeida e Rijo (2011), porém os dados observados são as resistividades aparentes e fases (simulados a partir da modelagem direta do MMT 2D (modos TM e TE) e acrescidos de ruídos). O processo de minimização da referida função objetivo é baseado no método de Marquardt (Press et al., 1992), juntamente 
com a estratégia proposta por Medeiros e Silva (1996) e Luiz (1999), que se trata da introdução de informação a priori (para o presente problema de inversão é especificamente chamada de introdução de vínculos absolutos). Para o maior entendimento tanto do método de Marquardt, como do algoritmo aqui aplicado na minimização da função objetivo e do seu critério de parada, recomendamos Almeida e Rijo (2011).

O algoritmo de inversão proposto por Almeida (2017), além de conter a primeira introdução de informação a priori, a saber, a introdução de vínculos absolutos, lhe é também acrescentado uma segunda introdução de informação a priori, a qual é justificada e incluída no referido algoritmo, como pode ser melhor compreendido a seguir.

A justificativa inicial é que na prospecção de hidrocarbonetos em águas profundas e ultra-profundas, os estratos geológicos de baixa resistividade elétrica são, geralmente, dominados por folhelhos, arenitos, siltitos e outras variações de rochas sedimentares com resistividade elétrica em torno de 1,0 Ohm-m, já os estratos geológicos com alta resistividade elétrica são, geralmente, as rochas reservatórios com hidrocarbonetos e rochas salinas com resistividade elétrica, respectivamente, entre 10,0 e $200,0 \mathrm{Ohm}-\mathrm{m}$ e entre 25,0 e 500,0 Ohm-m (Hoversten et al., 2000; Rijo, 2007; Pinto, 2009). Assim, a partir dessas informações a priori, é proposto, neste trabalho, que os parâmetros de resistividades elétricas estimados possuam resistividades no intervalo entre 0,85 e 250,0 Ohm-m (o qual é aqui denotado por intervalo de resistividades elétricas a priori). A segunda justificativa é que, por outro lado, os parâmetros de resistividades elétricas estimados durante o processo de inversão, podem apresentar amplitudes sub ou super estimadas, o que por sua vez, podem ocasionar instabilidade numérica durante o cálculo das respostas advindas do algoritmo de modelagem direta do MMT 2D, modelagem esta que é utilizada em algumas etapas do algoritmo de inversão do MMT 2D aqui proposto. Assim, para evitar esta instabilidade numérica, se faz necessária a introdução do proposto intervalo de resistividade elétrica a priori no presente algoritmo de inversão (Almeida, 2017).

A inclusão do referido intervalo a priori, no algoritmo presente, se faz da seguinte maneira: logo após o cálculo das resistividades elétricas estimadas (na iteração atual) é verificado qual entre estas resistividades elétricas que não pertencem ao referido intervalo a priori, e assim estas resistividades elétricas estimadas (as que não pertencentes ao intervalo a priori) deixam de apresentar suas atuais amplitudes e/ou valores de resistividades para voltarem a apresentar suas amplitudes de resistividades da iteração anterior (Almeida, 2017).

\section{Modelo proposto e os dados observados sintéticos}

Inicialmente apresentamos um modelo geoelétrico em um ambiente altamente resistivo proveniente da prospecção de hidrocarbonetos em águas profundas com características das bacias brasileiras offshore. Tal modelo é submetido à inversão geofísica, através dos dados observados do MMT 2D segundo os modos TM, TE e os dados conjuntos dos modos TM e TE , aplicando a minimização da função objetivo proposta por Almeida e Rijo (2011), a qual é minimizada pelo método de Marquardt (Press et al., 1992).

O modelo geoelétrico MMT 2D segundo características altamente resistivas em águas profundas (Almeida, 2017), (Fig.1), é expresso pela seção segundo a perspectiva do plano $z \times x$. Onde a camada de sal apresenta uma largura na direção $x$ de $24 \mathrm{~km}$, uma espessura $h_{3}$ igual a $2000 \mathrm{~m}$ e uma resistividade elétrica $\rho_{3}$ igual a 100 Ohm-m. A camada que representa o reservatório com hidrocarbonetos possui uma largura na direção $x$ de $20 \mathrm{~km}$, uma espessura $h_{4}$ igual $1500 \mathrm{~m}$, uma profundidade do assoalho marinho de $3000 \mathrm{~m}$ e apresenta uma resistividade $\rho_{4}$ igual a 50 Ohm-m.

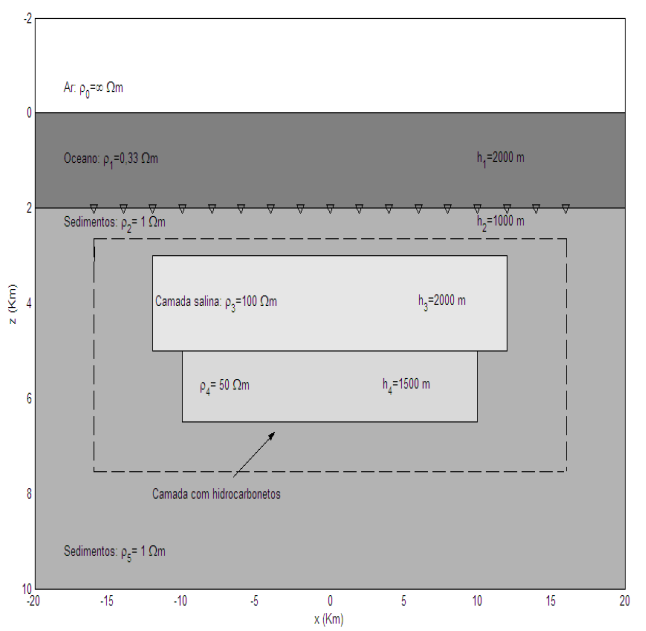

Figura 1 - Seção no plano $z \times x$ referente ao modelo geoelétrico 2D com camada salina e reservatório com hidrocarbonetos.

Observa-se na Fig.1 os receptores localizados e afixados no assoalho marinho, os quais são caracterizados pelo símbolo $\nabla$ e apresentam um espaçamento de $2000 \mathrm{em}$ 2000 metros, totalizando 17 receptores (pontos de sondagens) do MMT, cobrindo uma extensão de 32 quilômetros, com cada receptor operando em 14 períodos distintos entre $10^{0}$ e $10^{4}$ segundos com distribuição uniforme em escala logarítmica na base dez.

Para aplicar a inversão geofísica referente ao modelo da Fig. 1, foram realizadas as simulações através das modelagens diretas do MMT 2D tanto segundo o modo TM quanto o modo TE sobre o modelo geoelétrico da Fig. 1 , com finalidade de se obter as resistividades aparentes e fases tanto para o modo TM quanto para o modo TE do 
MMT 2D. Sendo as resistividades aparentes acrescidas com $7 \%$ de ruído e as fases acrescidas com $3 \%$ de ruído, criando assim os dados observados sintéticos aplicados posteriormente na inversão geofísica do MMT 2D.

São apresentadas nas Figs. 2 e 3, abaixo, a pseudoseção de resistividades aparentes (em Ohm-m) e a seção de fases da impedância (em graus), respectivamente, segundo o modo TM, referentes ao modelo geoelétrico do MMT 2D (Fig.1). Estas seções apresentam os períodos em escala logarítmica na base dez, e estão associadas à seção geoelétrica no plano $z \times x$ apresentada na Fig.1.

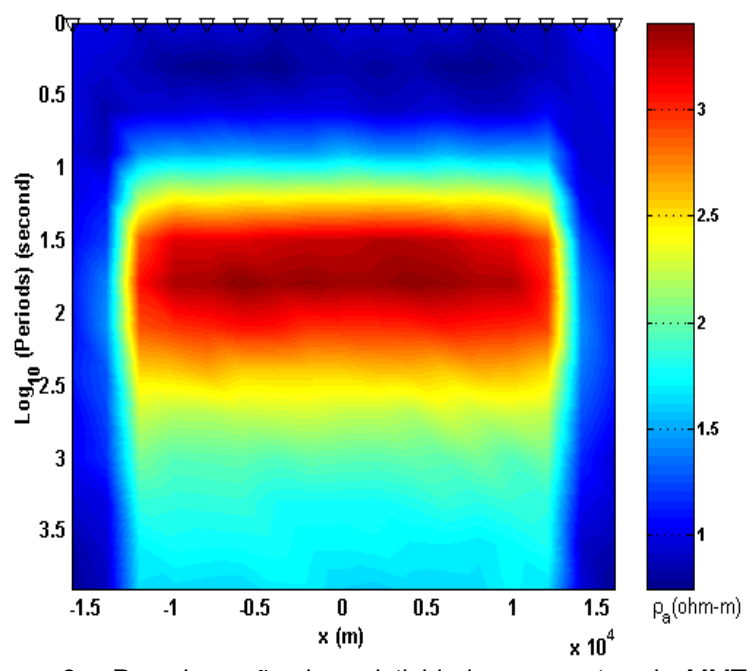

Figura 2 - Pseudo-seção de resistividades aparentes do MMT 2D segundo o modo TM referente ao modelo geoelétrico da Fig.1.

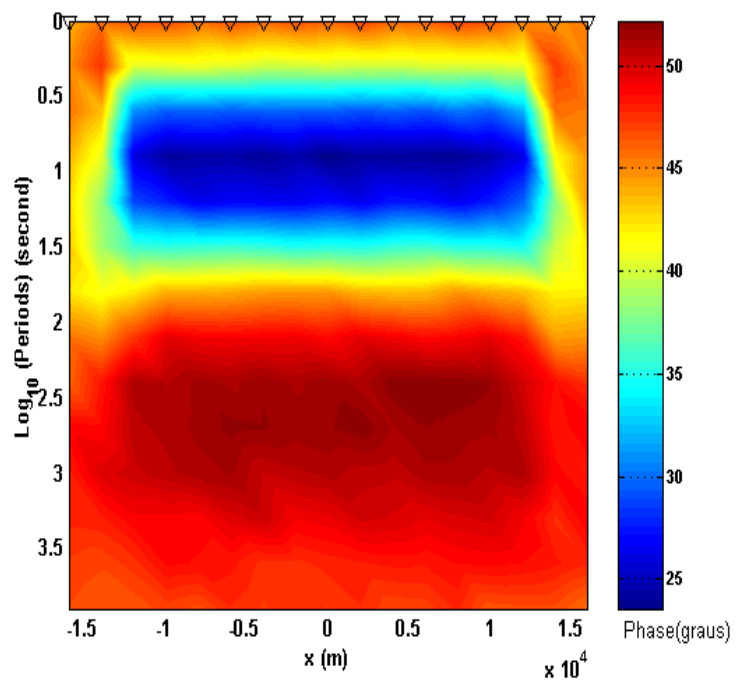

Figura 3 - Seção de fase da impedância do MMT 2D segundo o modo TM referente ao modelo geoelétrico da Fig.1.
Finalmente são apresentadas nas Figs. 4 e 5 a pseudoseção de resistividades aparentes (em Ohm-m) e a seção de fases da impedância (em graus), respectivamente, segundo o modo TE, referentes ao modelo geoelétrico do MMT 2D (Fig.1). Estas seções também apresentam os períodos em escala logarítmica na base dez, e estão associadas à seção geoelétrica no plano $z \times x$ apresentada na Fig.1.

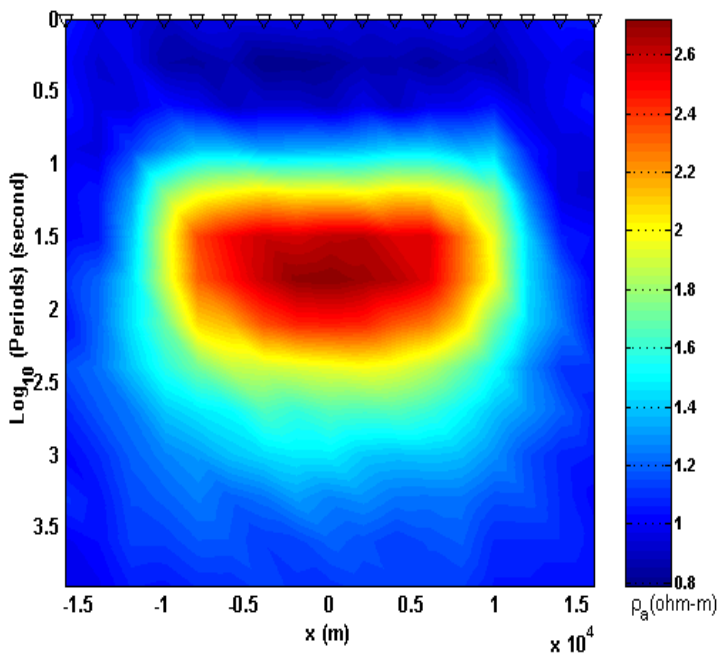

Figura 4 - Pseudo-seção de resistividades aparentes do MMT 2D segundo o modo TE referente ao modelo geoelétrico da Fig.1.

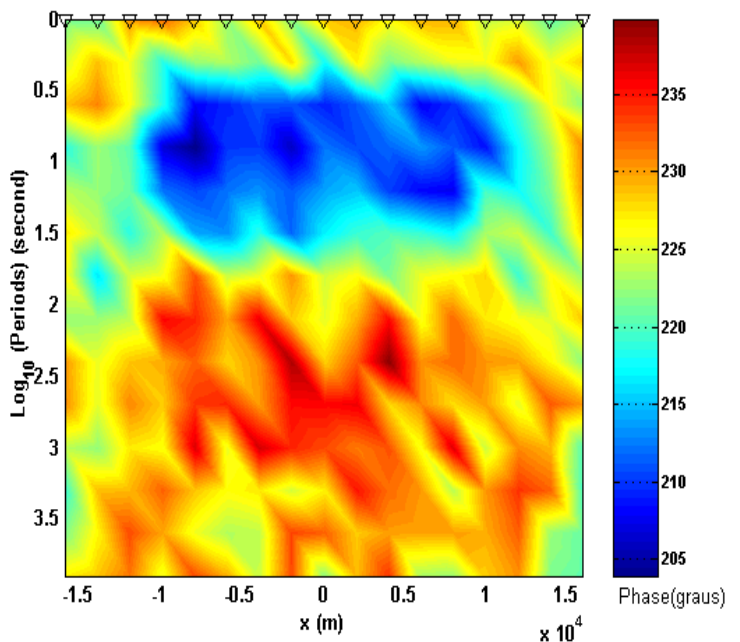

Figura 5 - Seção de fase da impedância do MMT 2D segundo o modo TE referente ao modelo geoelétrico da Fig.1. 


\section{Resultados de inversão}

Observa-se na Fig.1 uma área delimitada por um retângulo tracejado, esta área é a região de interesse para a inversão geofísica de dados do MMT 2D, segundo os modos TM e TE. Na prática a região de interesse pode ser delimitada através da interpretação de seções sísmicas em profundidade e, em particular quanto a sua extensão, pelas pseudo-seções de resistividades aparentes Figs. 2 e 4 . A região de interesse proposta é dividida em células retangulares, menores, às quais apresentam uma determinada amplitude de resistividade elétrica durante o processo de inversão, porém para o início de cada processo de inversão (MMT 2D modos TM e TE) foi admitido o valor de 1,0 Ohm-m para cada célula. Totalizando para cada processo de inversão, tanto através do modo TM, quanto pelo modo TE e finalmente pelos dados conjuntos dos modos TM e TE, 176 células de resistividades elétricas. Entre estas 176 células, 16 células são vinculadas através de vínculos absolutos, isto é, para cada uma destas 16 células admitimos amplitudes fixas de resistividades elétricas iguais a 1,0 Ohm-m, em que estas células vinculadas estão localizadas a uma profundidade entre 2250 e 2500 metros em relação à superfície do oceano e abrangem uma extensão de 32 quilômetros.

Comum aos três processos de inversão do método MMT 2D: modo TM, modo TE e dados conjuntos dos modos TM e TE, são as amplitudes para os parâmetros de Marquardt e de Lagrange, às quais foram admitidas de forma empírica, isto é, para o parâmetro de Marquardt foi admitido um valor igual a 1,0 e para o parâmetro de Lagrange foi admitido um valor positivo, os quais propiciaram a minimização das funções objetivos, com consequente convergência dos parâmetros de resistividades estimados. Assim, para um dado valor de tolerância, da norma do máximo aplicada sobre o vetor resultante da diferença entre dois vetores de iterações consecutivas dos parâmetros de resistividades elétricas estimados, donde se chegou ao valor de 50,0 para o parâmetro de Lagrange sobre a inversão do MMT 2D, uma vez dado o valor de tolerância para a norma do máximo igual a $10^{-5}$.

As três inversões foram calculadas em uma máquina com CPU 2,83 GHz, 3,46 GB de RAM e administrado por um Sistema Operacional Windows XP Professional. Dessa forma, cada processo de inversão teve duração média de 29 horas.

Apresentamos a seguir os resultados de inversão utilizando os dados 2D do MMT através do modo TM (Fig.6), do modo TE (Fig.7) e dos dados conjuntos dos modos TM e TE (Fig.8) e/ou dados conjuntos 2D dos modos TM e TE do MMT. Estes resultados são apresentados em termos da resistividade elétrica (Ohm$\mathrm{m})$. Para uma melhor distinção entre o meio mais resistivo (rochas salinas e rochas reservatório com hidrocarbonetos) e o meio menos resistivo (rochas sedimentares como folhelhos, siltitos, arenitos e outros) apresentamos as amplitudes das resistividades elétricas em escala logarítmica na base dez.

Inicialmente, abaixo, apresentamos os resultados de inversão utilizando os dados do MMT 2D através do modo TM (Fig.6) e do modo TE (Fig.7), em termos da resistividade elétrica $(\mathrm{Ohm}-\mathrm{m})$.
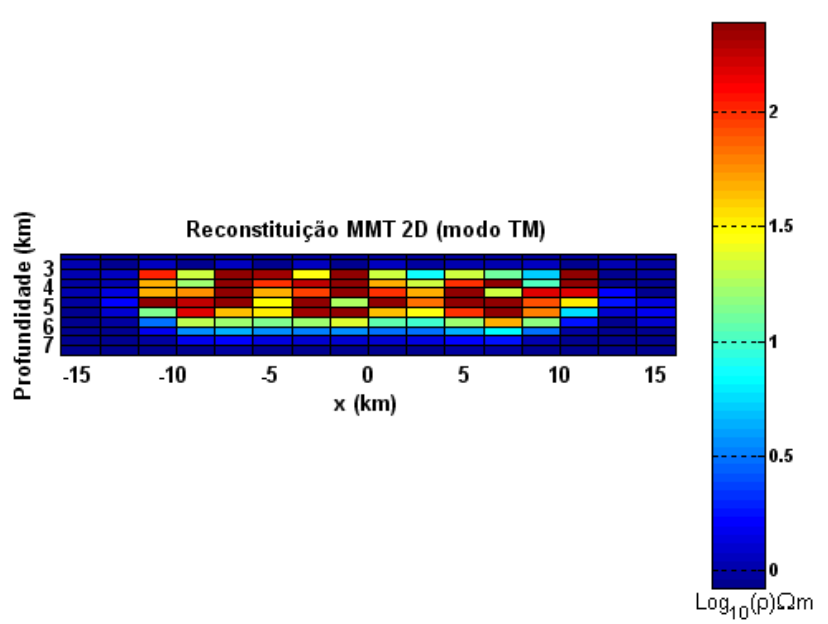

Figura 6 - Reconstituição do modelo geoelétrico 2D (Fig.1) através das resistividades aparentes (Fig.2) e das fases de impedância (Fig.3) do MMT 2D (modo TM) em termos da resistividade elétrica $(\mathrm{Ohm}-\mathrm{m})$.

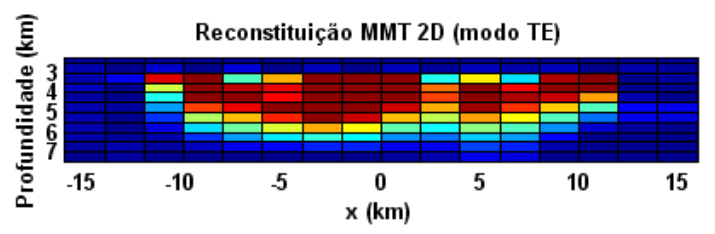

Figura 7 - Reconstituição do modelo geoelétrico 2D (Fig.1) através das resistividades aparentes (Fig.4) e das fases de impedância (Fig.5) do MMT 2D (modo TE) em termos da resistividade elétrica (Ohm-m).

Em ambos os resultados a cima, Figs.6 e 7, é possível observar a distinção do meio menos resistivo (em azul escuro) em relação ao meio mais resistivo (em laranja avermelhado). Nestes resultados, é possível localizar 
tanto o topo quanto a base do corpo mais resistivo (laranja avermelhado), assim como sua extensão, resultando em um bom delineamento do meio mais resistivo em relação ao meio menos resistivo das rochas sedimentares encaixantes (azul escuro).

Finalmente, abaixo, apresentamos o resultado de inversão utilizando os dados conjuntos do MMT 2D através dos modos TM (Fig.6) e TE (Fig.7) e/ou dados conjuntos dos modos TM e TE do MMT 2D, em termos da resistividade elétrica (Ohm-m).

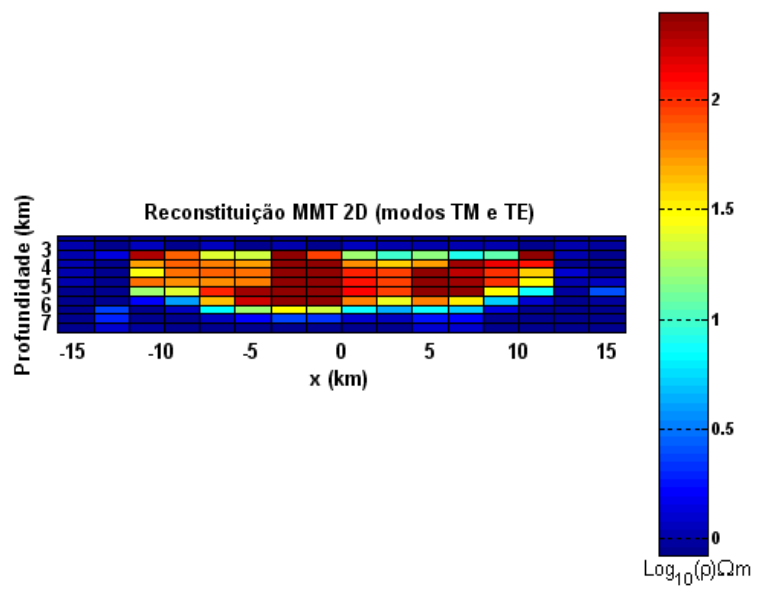

Figura 8 - Reconstituição do modelo geoelétrico 2D (Fig.1) através das resistividades aparentes (Figs.2 e 4) e das fases da impedância (Figs.3 e 5) do MMT 2D (dados conjuntos dos modos TM e TE do MMT 2D) em termos da resistividade elétrica (Ohm-m).

Observa-se na Fig.8 uma melhor uniformidade na distribuição da resistividade elétrica do corpo mais resistivo, que varia entre o laranja avermelhado e o vermelho escuro, em relação às resistividades elétricas do corpo mais resistivo das Figs. 6 e 7 com tonalidades alaranjadas, avermelhadas e amarela esverdeadas, com consequente melhor delineamento do corpo mais resistivo em relação às rochas sedimentares menos resistivas em azul escuro. Este resultado ocorre pela inclusão, adequada, de dados observados ao processo de inversão, o qual propicia uma melhor aproximação da real distribuição de resistividade elétrica absoluta em subsuperfície.

\section{Discussão e Conclusões}

$\mathrm{Na}$ fig. 8 é possível observar um melhor delineamento e uniformidade na distribuição das resistividades elétricas do corpo mais resistivo (laranja avermelhado) em relação aos corpos mais resistivos das figs. 6 e 7 . Isto ocorre graças ao adequado acréscimo de dados observados ao processo de inversão.

A metodologia de inversão proposta por Almeida e Rijo (2011) aplicada na inversão $2 \mathrm{D}$ de dados do MMT (modos TM e TE), presente neste trabalho, à qual inclui informação a priori de vínculos absolutos e a introdução do intervalo real de resistividades elétrica a priori, proposta por Almeida (2017), mostra-se eficaz, uma vez que nas figs. 6-8 é possível distinguir a base, o topo e a extensão do corpo mais resistivo em relação às rochas encaixantes sedimentares menos resistivas.

Nos três resultados acima, Figs. 6-8, não é possível distinguir entre o corpo salino e corpo reservatório com hidrocarbonetos de acordo com o modelo geoelétrico bidimensional (Fig.1). Isto se dá, devido tanto ao corpo salino quanto ao corpo com hidrocarbonetos possuírem altas resistividades elétricas, resultando em um "baixo" contraste e/ou razão de resistividade elétrica.

\section{Agradecimentos}

O autor agradece ao Conselho da Faculdade de Matemática e também ao Conselho do Campus Universitário de Castanhal da Universidade Federal do Pará pela aprovação do projeto de pesquisa, que a partir do qual foi possível o desenvolvimento deste trabalho.

\section{Referências}

Almeida, F. L., 2017. Inversão 2D de dados MMT de Ambientes Altamente Resistivos em Águas Profundas. $15^{\text {th }}$ International Congress of the Brazilian Geophysical Society held in Rio de Janeiro, Brazil. 1 CD-ROM.

Almeida, F. L., 2015. Modelagem e Análise de dados MMT 1D e 2D segundo Ambientes Altamente Resistivos em Águas Profundas. $14^{\text {th }}$ International Congress of the Brazilian Geophysical Society held in Rio de Janeiro, Brazil. 1 CD-ROM.

Almeida, F.L, Rijo, L., 2011. Modelagens Direta e Inversa de Dados mCSEM 3D. RBGf. v.29(2), p. 289-308.

Becker, E. B., Carey, G. F., Oden, J. T. -1981- Finit elements - An introduction. New Jersey: Prentice-Hall, $258 p$.

Luiz, J. G., 1999. Informação a priori na inversão de dados Magnetotelúricos. Tese de Doutorado, UFPA. 92 p.

Hoversten, G. M., Constable, S. C., Moooison, H. F., 2000. Marine magnetotellurics for base-of-salt mapping: Gulf of Mexico field test at the Gemini structure. Geophysics, n. 5, p. 1476-1488.

Medeiros, W. E. \& Silva, J. B. C., 1996. Geophysical inversion using approximate equality constrains. Geophysics, vol. 61 , p. $1678-1688$.

Pinto, V. R., 2009. O Método Magnetotelúrico Marinho (MMT) na Exploração de Hidrocarbonetos. Dissertação de Mestrado, ON, $63 \mathrm{p}$. 
Press, W. H., Teukolsky, S. A., Vetterling, W. T. and FIAannery, B. P.,1992. Numerical Recipes in Fortran 77. Cambridge University Press. 2nd ed, p. 678-680.

Rijo, L., 2002. Teoria dos Métodos Eletromagnético I, II e III - Notas de aula. Departamento de Geofísica, UFPa.

Rijo, L., 2007. Cooperação Universidade-Indústria: O caso do método marine Controlled Source Electromagenetic (CSEM) na exploração de hidrocarbonetos. $10^{\text {th }}$ International Congress of the Brazilian Geophysical Society held in Rio de Janeiro, Brazil. 1 CD-ROM.

Saito, K. K. S., 2009. Modelagem Computacional de Dados Magnetotelúricos Marinhos 2-D. Dissertação de Mestrado, UFPA, $46 \mathrm{p}$.

Souza, V. C. T., Rijo, L. e Silva, M. W. C., 2005. The preconditioned biconjugate gradient algorithm applied to geophysical electromagnetic modeling. $9^{\text {th }}$ International Congress of the Brazilian Geophysical Society held in Salvador, Brazil. 